\title{
Podoserpula miranda nom prov., une nouvelle espèce de champignon très spectaculaire découverte en Nouvelle-Calédonie
}

Marc Ducousso ${ }^{1,2}$

Sylvie Proust ${ }^{2}$

Denis VIGIER ${ }^{2}$

Guillaume EYSSARTIER ${ }^{3}$

${ }^{1}$ Laboratoire des symbioses tropicales et méditerranéennes

Umr 113 Agro-M/Cirad/Inra/Ird/Um2 et Institut agronomique néo-calédonien

BP A5, 98848 Nouméa Nouvelle-Calédonie

2 Société mycologique de Nouvelle-Calédonie BP A5, 98848 Nouméa Nouvelle-Calédonie

3 Société mycologique de France 250, étangs de Béon 45210 Bazoches-sur-le-Betz

France
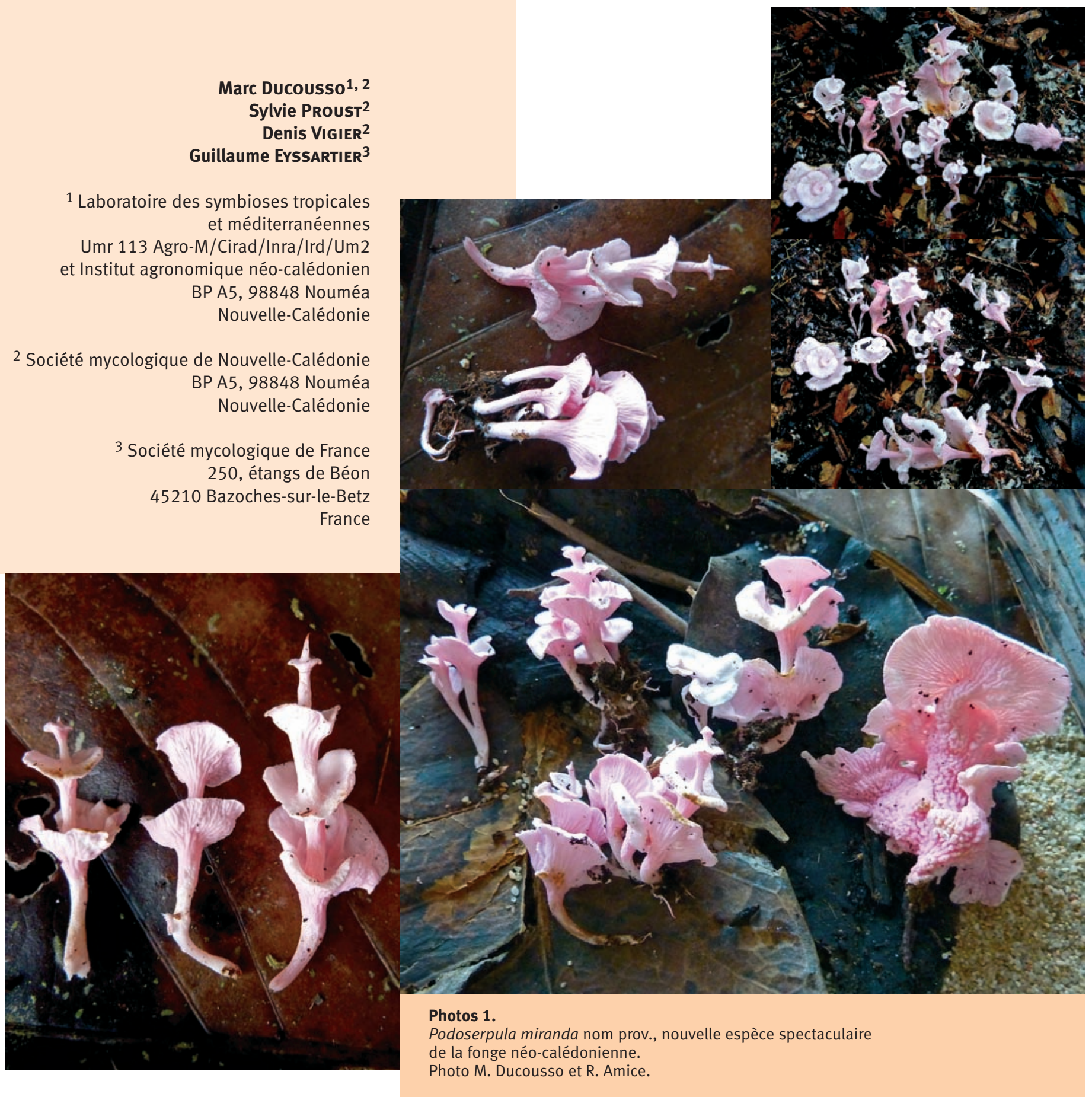


\section{RÉSUMÉ}

PODOSERPULA MIRANDA NOM

PROV., UNE NOUVELLE ESPÈCE

DE CHAMPIGNON TRÈS SPECTACULAIRE DÉCOUVERTE EN NOUVELLE-CALÉDONIE

La Nouvelle-Calédonie est une île du Pacifique sud reconnue comme un " hotspot» de biodiversité. Toutefois, l'inventaire des champignons de ce territoire de $17000 \mathrm{~km}^{2}$ est encore très fragmentaire ; à peine $1 \%$ des espèces auraient été décrites à ce jour ! Le récent développement d'un programme de recherche sur le rôle des microorganismes symbiotiques, notamment les champignons, dans l'adaptation des plantes aux sols extrêmes et la création de la Société mycologique de Nouvelle-Calédonie ont permis l'identification de plusieurs centaines d'espèces nouvelles pour ce territoire, mais aussi la découverte de nouvelles espèces, dont le spectaculaire Podoserpula miranda nom prov.

Mots-clés : Podoserpula, espèce, flore fongique, Nouvelle-Calédonie.

\section{ABSTRACT}

\section{PODOSERPULA MIRANDA PROV. NAME, A SPECTACULAR NEW FUNGUS SPECIES DISCOVERED IN NEW-CALEDONIA}

Although New Caledonia, a 17,000 km² island in the South Pacific, is recognised as a biodiversity hot-spot, the inventory of New Caledonian fungi is far from complete: it is estimated that barely $1 \%$ of fungal species found in New Caledonia have been described to date. Thanks to a research programme on the role of mycorrhizal fungi in plant adaptation to extreme soil conditions, and to the creation of the Mycological Society for New Caledonia, several hundred species have been identified and new species have been discovered, including the remarkable Podoserpula miranda prov. name.

Keywords: Podoserpula, species, fungus flora, New Caledonia.

\section{RESUMEN}

\section{PODOSERPULA MIRANDA NOMBRE PROV., UNA NUEVA ESPECIE DE HONGO MUY LLAMATIVA DESCUBIERTA EN NUEVA CALEDONIA}

Nueva Caledonia es un archipiélago del Pacífico Sur considerado como un «hotspot» de biodiversidad. No obstante, el inventario micológico de este territorio de $17000 \mathrm{~km}^{2}$ aún sigue siendo muy parcial y, hasta la fecha, sólo se habría descrito el 1\% de las especies. El reciente desarrollo de un programa de investigación sobre el papel de los microorganismos simbióticos, especialmente los hongos, en la adaptación de las plantas a suelos extremos, así como la creación de la Société Mycologique de Nouvelle-Calédonie, no sólo han permitido la identificación de varios centenares de especies nuevas para este territorio, sino también el descubrimiento de nuevas especies como el llamativo Podoserpula miranda nombre prov.

Palabras clave: Podoserpula, especie, flora micológica, Nueva Caledonia. 


\section{Un parmi trente mille}

La Nouvelle-Calédonie est une île située dans l'océan Pacifique Sud à 1200 km à l'est de l'Australie. De par sa position géographique particulière, cette île a été découverte très tardivement par James Cook en 1774. Depuis cette date, des missions scientifiques visant à développer les connaissances des milieux physique, biologique et humain se sont succédé pour aboutir à l'installation permanente de scientifiques sur ce territoire. Au plan biologique, l'ensemble des travaux a permis de classer la Nouvelle-Calédonie comme « hotspot» mondial de biodiversité (MYers et al., 2000). Toutefois, les travaux menés en mycologie sont encore très fragmentaires. En effet, une récente estimation du nombre de champignons fait état de 30000 espèces en Nouvelle-Calédonie (EysSARTIER, 2009), alors qu'aujourd'hui à peine plus de 300 espèces y ont été décrites (HORAK, MouchaccA, 1998) ; 99 \% des espèces restent donc inconnues des scientifiques ! Depuis 2006, une équipe de l'Umr ${ }^{1} 113$, Laboratoire des symbioses tropicales et méditerranéennes, développe, au sein du centre $\mathrm{Ird}^{2} \mathrm{de}$ Nouméa et en partenariat avec l'Institut agronomique néo-calédonien, des recherches sur le rôle des champignons et des bactéries dans l'adaptation des plantes aux sols si particuliers de la Nouvelle-Calédonie. En effet, ces sols sont constitués majoritairement de fer (50 à 85 \%), mais sont également très riches en métaux lourds potentiellement toxiques (nickel, chrome, magnésium, cobalt), très pauvres en nutriments (azote, potassium, phosphore) et avec un rapport calcium/magnésium $(\mathrm{Ca} / \mathrm{Mg})$ pouvant être inférieur à $10^{-2}$. Ces conditions qui ont permis l'émergence d'une flore très originale endémique à $80 \%$ ont également permis l'émergence d'une flore fongique, elle aussi très originale. Ainsi, en moins de quatre ans, les mycologues de Nouvelle-Calédonie ont réuni un herbier mycologique de plus de 2000 spécimens et collaborent activement avec le Muséum national d'histoire naturelle de Paris à la description des espèces néo-calédoniennes (EYSSARTIER, 2009). La récente prise de conscience de l'originalité des champignons de Nouvelle-Calédonie a été un élément qui a conduit des amateurs passionnés à fonder une association, la Société mycologique de Nouvelle-Calédonie (Smnc) ${ }^{3}$, ayant pour objet, entre autres, de produire des connaissances sur les champignons de la Nouvelle-Calédonie. C'est à l'occasion d'une sortie mycologique organisée par la Smnc que les sociétaires présents ont pu admirer une nouvelle espèce, certainement parmi les plus spectaculaires de la mycologie (photo 1). La principale caractéristique qui rend cette espèce originale est son architecture où des chapeaux s'empilent, jusqu'à six, les uns au-dessus des autres pour ne former qu'un champignon de huit à dix centimètres de hauteur. Les couleurs rose vif de l'hyménium et rose légèrement plus pâle du reste du champignon (pied et chapeau) contribuent à faire de cette espèce grégaire un champignon tout à fait exceptionnel.

Le seul champignon connu à ce jour pour former des empilements autour d'un axe central appartient au genre monospécifique Podoserpula (REID, 1963). Toutefois, des différences importantes ont été notées entre l'espèce néo-calédonienne et Podoserpula pusio (Berk.) D.A. Reid. D’abord sur le plan de l'architecture : les chapeaux de $P$. pusio présentent des étages presque horizontaux et souvent excentrés de l'axe porteur.

\footnotetext{
1 Unité mixte de recherche.

2 Institut de recherche pour le développement.
}

Par ailleurs, la chair de $P$. pusio est relativement épaisse. Pour l'espèce néo-calédonienne à la chair blanche très fine, les étages ressemblent à de petits champignons en forme d'entonnoir au centre duquel un autre champignon de même forme, mais plus petit, pousse et ainsi de suite jusqu'à cinq ou six fois. Ainsi, le champignon qui mesure jusqu'à dix centimètres est centré sur son axe. Sur le bord supérieur d'étage de certains spécimens, on remarque parfois le début de la formation d'un étage, mais seul l'étage situé au centre se développe complètement pour former l'étage supérieur. Les parties fertiles ou hyménium en replis lamellaires, comme les craterelles, sont d'un rose vif très caractéristique, alors que $P$. pusio est entièrement crème à crème de teinte ocre ou jaune orangé pâle.

Une autre différence écologique d'importance est la capacité de l'espèce néo-calédonienne à former une association symbiotique ectomycorhizienne avec l'espèce dominante de la canopée, Arillastrum gummiferum (Brongn. et Gris) Pancher ex-Baill., appelée localement "chêne gomme ". De son côté, $P$. pusio est une espèce saprophyte qui se développe sur le bois mort en décomposition.

Malgré la variabilité importante notée dans la forme de $P$. pusio, l'ensemble de ces différences et de ces ressemblances permet aujourd'hui de classer ce champignon dans le genre Podoserpula ; il permet également d'être certain qu'il s'agit d'une espèce nouvelle pour la science. Compte tenu de l'étonnement suscité par ce champignon et de l'émerveillement de ses découvreurs, l'épithète spécifique miranda sera proposée pour nommer l'espèce.

Bien qu'appartenant à un groupe de champignons ne comprenant pas a priori d'espèces toxiques, en raison de son odeur de radis rose et de sa texture fibro-cotonneuse, il est très peu probable que ce champignon ait un quelconque intérêt gastronomique.

La découverte de cette espèce est une étape qui marquera la mycologie néo-calédonienne qui n'en est qu'à ses débuts ; il ne reste plus qu'à décrire 29699 espèces en NouvelleCalédonie !

\section{Bibliographie}

EYSSARTIER G., 2009. Mission d'introduction à un inventaire mycologique de Nouvelle-Calédonie. Rapport de mission Cirad, mars-avril 2009, 10 p.

HORAK E., MOUCHACCA J., 1998. Annotated checklist of New Caledonian Basidiomycota. I. Holobasidiomycetes. Mycotaxon, $68: 75-129$.

MYERS N., MITTERMEIER R. A., MITTERMEIER C. G., DA FONSECA G. A. B., KENT J., 2000. Biodiversity hotspots for conservation priorities. Nature, $403: 853-858$.

REID D. A., 1963. Fungi Venezuelani. VI (New or interesting records of Australasian basidiomycetes: IV). Podoserpula Reid : a new genus based on Craterellus pusio Berk. (C. multiplex Cooke \& Massee). Kew Bulletin, 16 : 437-445.

\footnotetext{
${ }^{3}$ Société mycologique de Nouvelle-Calédonie ; site web consultable
} à l'adresse suivante : www.smnc.nc. 